\title{
2. Über die Strahlung des Lichtbogens ${ }^{\text {) }}$; von Wilhelm Hallwachs.
}

\section{\& 1. Einleitung.}

Die sichtbare Strahlung der Bogenlampe geht wesentlich vom Krater aus, nur außerordentlich wenig trägt zu ihr der Bogen selbst bei. Am schärfsten tritt diese Tatsache in der starken Abhängigkeit der Strahlungsintensität von der Richtung hervor: $40-50^{\circ}$ unter die Horizontale sendet die Gleichstromlampe mit vertikalen Kohlen etwa 5-6 mal soviel Licht wie nach der Horizontalen. Unter diesen Umständen läßt sich über die Eigenschaften, z. B. das Strahlungsgesetz des Bogens selbst, des vielleicht jetzt physikalisch interessantesten Teiles der Bogenlampe, aus Beobachtungen der sichtbaren Strahlung nichts ermitteln.

Es fragt sich, ob für die ultraviolette Strahlung z. B. den Teil derselben, welcher lichtelektrisch am wirksamsten ist, und welcher kurz die lichtelektrische Strahlung heißen möge, die Verhältnisse ebenso liegen. Soweit ich mich durch die Literatur und mündliche unterrichten konnte, scheint die allgemeine Meinung dahin zu gehen, daB man bei lichtelektrischen Versuchen mit der Bogenlampe wegen der Abhängigkeit von der Richtung auf scharfe Orientierung sehen müsse, weil vom Krater her ein beträchtlicher Teil der ultravioletten Strahlung komme. Andererseits fand ich schon bei früheren Untersuchungen immer, daß die lichtelektrische Strahlung bei konstanter Stromstärke mit der Bogenlänge auBerordentlich stark zunimmt, was anderen Beobachtern, obwohl ich mich nach Angaben darüber in der Literatur vergeblich bemüht habe, vermutlich auch aufgefallen ist. Es steht dies im vollkommenen Gegensatz zum Verhalten der sichtbaren Strahlung, welche,

1) Für die erfolgreiche Hilfe, welche mir Hr. Dr. W. Ziegler, jetzt in Santiago (Chile), bei dieser Arbeit geleistet bat, möchte ich ibm auch an dieser Stelle meinen besten Dank aussprechen. 
wenn man von sehr kleinen Bogenlängen absiecht, mit wachsender Bogenlänge bei konstanter Stromstärke nicht zu, sondern etwas abnimmt. ${ }^{1}$ )

Die nähere Untersuchung dieser Verhältnisse ergab nun, wie im folgenden dargelegt werden soll, daB die lichtelektrische Strahlung sozusagen ausschließlich vom Bogen geliefert wird, dab vom Krater her kein, oder schärfer kein die Versuchsfehler, d. h. wenige Prozente, überschreitender Betrag derselben kommt. Dieses Resultat liefert dann einerseits ein Mittel um ein Strahlungsgesetz des Bogens, $d$. $h$. die Abhängigkeit der lichtelektrischen Strahlungsintensität von Strom und Spannung, aufzusuchen, andererseits führt $\theta$ s eine beträchtliche Erleichterung für experimentelle Arbeiten, welche diese Strahlung des Bogens benutzen, herbei, weil eine scharfe Orientierung der exponierten Objekte gegen die Bogenlampe wegen der groBen Unabhängigkeit der Lichtstärke von der Richtung unnötig wird. Alle folgenden Untersuchungen gelten für normal brennenden Bogen bei vertikalen Kohlen mit der Anode oben.

\section{§ 2. Unabhängigkeit der lichtelektriøchen strahlung von der Richtung.}

Zum Nachweis, daß die lichtelektrische Strahlung in weiten Grenzen unabhängig von der Richtung ist, diente folgende Anordnung. Zwei lichtelektrische Zellen standen in verschiedenen Orientierungen zur Lampe, z. B. die eine in der Horizontalen durch den Bogen, die andere weit unter der Horizontalen, so daB die auf sie fallenden Strahlen z. B. einen Winkel von $40^{\circ}$ mit der Horizontalen bildeten. Nachdem das Verbältnis der lichtelektrischen Empfindlichkeit der beiden Zellen in diesen Stellungen ermittelt war, erhielten dieselben dicht nebeneinander Aufstellung, worauf wieder jenes Verhältnis bestimmt wurde. Der zweite Versuch liefert das Verbältnis der Zellenkonstanten, mit dessen Hilfe sich dann aus dem ersten das Verhältnis der Lichtstärken in den beiden Richtungen ergibt.

1) A. Blondel, Ecl. Electr. 10. p. 289, 496, 539. 1897. Mr. Ayrton in Mrs. Ayrton, „Electric Arc", London, The electrician p. 396. 1903; beides nach letzterem Werk zitiert. 
Die verwendeten lichtelektrischen Zellen bestanden aus einem als Träger dienenden Zinkblech, $14 \times 4 \mathrm{~cm}$, auf dem parallel davor, in etwa 6-10 $\mathrm{mm}$ Abstand, ein etwa ebenso groBes Drahtnetz mittels Schellacksäulchen befestigt war. Die auf ihren lichtelektrischen Verlust zu untersuchenden Platten ließen sich in diese Vorrichtung einschieben und auf dem Zinkblech flach liegend mit einer Schraube befestigen. Die Zelle hing dabei an einem am Zinkblech befestigten Schellackstab. Das Drahtnetz, auf welches die Strahlen der Lampe zunächst fielen, stand mit einer andererseits geerdeten Batterie kleiner Akkumulatoren von +280 Volt dauernd in Verbindung, während rom Zinkblech eine durch Schellackstützen isolierte Leitung zu einem Al-Elektroskop führte, dessen Hülle an Erde lag. Mittels Fernrohres wurde die Stellung der von hinten beleuchteten Blättchen auf einer in die Deckplatte des Elek. troskops eingeätzten Teilung abgelesen. Nach Ableitung des Elektroskops bei abgeblendeter Bogenlampe gelangte die Zeit $\boldsymbol{r}$ zur Beobachtung, welche vom Entfernen der Blende bis zum Anwachsen der Divergenz der Blättchen auf einen für alle Versuche gleichen Betrag, bestimmten Teilstrich des Elektroskops, verstrich. Dieser Betrag entsprach etwa $2 / 3$ der oben genannten Batteriespannung. Der reziproke Wert von $\tau$ gibt ein $\mathrm{MaB}$ für die Intensität der lichtelektrischen Strahlung der Bogenlampe.

Um die Beobachtungen nicht durch die raschen Änderungen unsicher zu machen, welche blanke Metallplatten hinsichtlich ihrer lichtelektrischen Emptindlichkeit erleiden, kamen in den Zellen Kupferoxydplatten ${ }^{1}$ ) zur Anwendung, welche, wie später gelegentlich gezeigt werden wird, verhältnismäßig konstant sind.

1) Auf die lichtelektrische Empfindlichkeit von CuO-Platten habe ich schon Götting. Nachr. 1889. Nr. 12; Wied. Ann. 37. p. 666, § 1. 1889 hingewiesen, um zu zeigen, dab die lichtelektrischen Erscheinungen nicht auf Oxydation und ähnliche chemische Ursachen zurückzuführen sind. Um die Mitte der 90 er Jahre habe ich gelegentlich eine größere Anzabl von Versuchen ausgeführt, welche alle zeigten, daß die lichtelektrischen Erscheinungen keine sekundären durch chemische Ursachen veranlaBten Phänomene bilden, wie damals vielfach angenommen wurde. Nachdem auch konstatiert worden war, daB kein Zusammenhang mit der Wasserfallelektrizität bestand, wie Hr. Thomson gemeint hatte, bewegten sich meine Versuche in der Richtung, zu untersuchen, ob durch die ultra- 
Die Bogenlampe war meistens eine Piette Kriřik-Differentiallampe von Schuckert. Um dieselbe, wie es die Versuche verlangten, auf möglichst feine Regulierung zu bringen, wurden die Sperrkegel mittels eines Korkes ausgehoben. Man muß dann allerdings in Kauf nehmen, daB die Lampe gelegentlich ins Pendeln kommt. Bei ausreichender Vorsicht läBt sich dies indessen genügend vermeiden und die Variationen der Spannung und damit der lichtelektrischen Strahlungsintensität bleiben viel kleiner wie beim Anlegen der für den gewöhnlichen Beleuchtungsgebrauch erforderlichen Sperrkegel. Die Lampe befand sich in einer großen Kiste, welche, mit nach außen führendem Abzugsrohr und entsprechendem Gegenloch versehen, den Austritt der Lampenprodukte in den Beobachtungsraum verhinderte. Im Laufe der Untersuchung ergab sich indessen, daß man wegen der Größe $\left(700 \mathrm{~m}^{3}\right)$ und guten Ventilation des Beobachtungsraumes auf das Abzugsrohr verzichten konnte.

Den Strom empfing die Lampe von einer Akkumulatorenbatterie, welche von anderen Anschlüssen, um die Lampe vor jeder Störung zu bewahren, frei blieb. Die Lampenspannung wurde mit einem Drehspulengalvanometer (Siemens \& Halske, Präzisionsinstrument), welches pro Volt 2 Skt. ergab, die Stromstärke mit einem Rapsschen Weicheiseninstrument laufend kontrolliert. Für gewöhnlich arbeitete man mit etwa $20 \mathrm{Amp}$, 49 Volt, $16 \mathrm{~mm}$ Docht- und $11 \mathrm{~mm}$ Vollkohle, die letzteren meist von Gebrüder Siemens. Die Zentrierung der Koblen geschah mit gröBter Sorgfalt und die Beobachtungen begannen erst dann, wenn die Kohlen gut eingebrannt waren, d. h. der Krater fehlerfreie Ausbildung gewonnen hatte, der Bogen symmetrisch saB und die negative Kohle keine verlängerte Spitze aufwies.

violette Belichtung irgend eine Strahlung ausgelöst werde. Durch die Fülle der Amtsgeschäfte in meiner früheren elektrotechnischen Professur nur selten zu eigener Arbeit frei, hoffte ich die Versuche nach meinem Wiedereintritt in die Physik abschlieBen zu können. Diese Hoffnung wurde durch die schönen Untersuchungen des Hrn. Lenard gegenstandslos. Das frühere Material zu veröffentlichen hat jetzt keinen $\mathrm{Zweck}$ mehr. Bei allen diesen Untersuchungen hat mir die im Vergleich zu anderen Substanzen verhältnismäBig große Konstanz des $\mathrm{CuO}$ gute Dienste geleistet. 
Mit der geschilderten Anordnung gelangten folgende Versuche zur Ausführung.

a) Vergleich der horizontalen mit der unter $40^{\circ}$ gegen die Horizontale geneigten Strahlung.

Zwei lichtelektrische Zellen I und II waren so aufgehängt, dab ihre Mittelpunkte von einer vom Bogen aus um $40^{\circ}$ unter die Horizontale geneigten Geraden getroffen wurden. Die beiden Zellen hingen direkt nebeneinander, der Abstand ihrer Mittelpunkte rom Bogen betrug $90 \mathrm{~cm}$. Durch den geringen Abstand von Platte und Netz bei den Zellen war eine merkbare gegenseitige Beeinflussung derselben vermieden, was Hilfsversuche bestätigten.

Von zwei CuO-Platten $g$ und $h$, kam erst $h$ nach I, $g$ nach II, wobei die beobachteten Ladungszeiten $\tau$ (vgl. p. 40) $h_{1}^{u}$ und $g_{\mathrm{II}}^{u}$ heilen sollen. Der Index $u$ weist darauf hin, dab die Platten $40^{\circ}$ unter der Horizontalen hingen. Sodann wurden die Platten vertauscht und die Ladungszeiten $h_{\mathrm{II}}^{u}$ und $g_{\mathrm{I}}^{u}$ erhalten. Zunächst möge ein Beispiel solcher Beobachtungen hier ausführlich folgen, zusammen mit den gleichzeitig abgelesenen Spannungen $P$ und Stromstärken $i$ :

Tabelle 1.

\begin{tabular}{|c|c|c|c|c|c|c|c|c|c|}
\hline $\begin{array}{c}P \\
\text { Volt }\end{array}$ & $\begin{array}{c}i \\
\text { Amp. }\end{array}$ & $\begin{array}{c}h_{\mathrm{I}}^{u} \\
\mathrm{sec}\end{array}$ & $\begin{array}{l}g_{\mathrm{II}}^{u} \\
\mathrm{sec}\end{array}$ & $\frac{h_{\mathrm{I}}^{u}}{g_{\mathrm{II}}^{u}}$ & $\begin{array}{c}P \\
\text { Volt }\end{array}$ & $\begin{array}{c}i \\
\text { A mp. }\end{array}$ & $\begin{array}{c}g_{\mathrm{I}}^{u} \\
\sec \end{array}$ & $\begin{array}{l}h_{\mathrm{II}}^{u} \\
\mathrm{sec}\end{array}$ & $\frac{g_{\mathrm{I}}^{u}}{h_{\mathrm{II}}^{q_{1}}}$ \\
\hline $\begin{array}{l}50,0 \\
49,5\end{array}$ & $\begin{array}{l}19,2 \\
18,9\end{array}$ & 33,5 & 45 & 0,745 & $\begin{array}{l}49,2 \\
50,2\end{array}$ & $\begin{array}{l}19,0 \\
18,5\end{array}$ & 34 & 45 & 0,757 \\
\hline $\begin{array}{l}50,5 \\
50,0\end{array}$ & $\begin{array}{l}18,8 \\
19,0\end{array}$ & 32 & 44 & 0,726 & $\begin{array}{l}49,8 \\
50,0\end{array}$ & $\begin{array}{l}18,5 \\
18,8\end{array}$ & 34,5 & 46 & 0,750 \\
\hline $\begin{array}{l}50,2 \\
50,5\end{array}$ & $\begin{array}{l}18,8 \\
18,5\end{array}$ & 32 & 45 & 0,711 & $\begin{array}{l}50,5 \\
49,2\end{array}$ & $\begin{array}{l}18,5 \\
19,5\end{array}$ & 35 & 47 & 0,745 \\
\hline Mittel : & $\frac{h_{\mathrm{I}}^{u}}{g_{\mathrm{dI}}^{u}}$ & & $\frac{g_{\mathrm{I}}^{u}}{h_{\mathrm{II}}^{u}}$ & 759 & $\begin{array}{l}50,5 \\
50,8\end{array}$ & $\begin{array}{l}18,5 \\
18,8\end{array}$ & 34 & 45 & 0,757 \\
\hline
\end{tabular}

Nach diesen Versuchen wurde Zelle I nahezu in der Horizontalen durch den Lichtbogen $\left(5^{0}\right.$ Neigung unter die Horizontale blieb noch) wieder in $90 \mathrm{~cm}$ Abstand mit ihren 
Platten vertikal aufgestellt, während II in der vorigen Lage verblieb. Es ergaben sich dann Ladungszeiten $g_{\mathrm{I}}^{o}$ und $h_{\mathrm{II}}^{u}$, bez. nach Vertauschung der Platten: $h_{\mathrm{I}}^{0}$ und $g_{\mathrm{II}}^{u}$ und nach abermaliger Vertauschung wieder $g_{\mathrm{I}}^{o}$ und $h_{\mathrm{II}}^{u}$. Die Tab. 2 gibt die gewonnenen Werte.

Tabelle 2.

\begin{tabular}{|c|c|c|c|c|c|c|c|c|c|}
\hline $\begin{array}{c}\dot{P} \\
\text { Volt }\end{array}$ & $\begin{array}{c}i \\
\text { Amp. }\end{array}$ & $\begin{array}{c}g_{1}^{o} \\
\text { sec }\end{array}$ & $\begin{array}{l}h_{11}^{n} \\
\sec \end{array}$ & $\frac{y_{\mathrm{I}}^{0}}{h_{\mathrm{II}}^{u b}}$ & $\begin{array}{c}P \\
\text { Volt }\end{array}$ & $\begin{array}{c}i \\
\text { Amp. }\end{array}$ & $\begin{array}{c}g_{\mathrm{I}}^{o} \\
\sec \end{array}$ & $\begin{array}{l}h_{\mathrm{II}}^{u} \\
\mathrm{sec}\end{array}$ & $\frac{g_{\mathrm{I}}^{o}}{h_{\mathrm{II}}^{u}}$ \\
\hline $\begin{array}{l}48,8 \\
49,0\end{array}$ & $\begin{array}{l}19,5 \\
19,8\end{array}$ & 30 & 50 & 0,600 & $\begin{array}{l}50,0 \\
49,8\end{array}$ & $\begin{array}{l}19,2 \\
19,0\end{array}$ & 30 & 52 & 0,577 \\
\hline $\begin{array}{l}49,0 \\
49,5\end{array}$ & $\begin{array}{l}19,5 \\
19,5\end{array}$ & 29 & 48 & 0,604 & $\begin{array}{l}49,8 \\
50,0\end{array}$ & $\begin{array}{l}18,5 \\
19,0\end{array}$ & 29,5 & 50 & 0,590 \\
\hline $\begin{array}{l}49,0 \\
49,8\end{array}$ & $\begin{array}{l}19,5 \\
19,2\end{array}$ & 29 & 48 & 0,604 & $\begin{array}{l}50,0 \\
50,2\end{array}$ & $\begin{array}{l}19,0 \\
19,0\end{array}$ & 29,5 & 50 & 0,590 \\
\hline & & $h_{\mathrm{I}}^{o}$ & $g_{\mathrm{II}}^{u}$ & $\begin{array}{c}h_{\mathrm{I}}^{o} \\
g_{\mathrm{II}}^{u} \\
\end{array}$ & $\begin{array}{l}50,0 \\
50,5\end{array}$ & $\begin{array}{l}18,8 \\
19,0\end{array}$ & 30 & 51,5 & 0,5582 \\
\hline $\begin{array}{l}49,5 \\
49,8\end{array}$ & $\begin{array}{l}19,2 \\
19,0\end{array}$ & 31 & 55 & 0,564 & & Mittel & \multirow{3}{*}{\multicolumn{3}{|c|}{$\begin{array}{l}g_{\mathrm{I}}^{o}: h_{\mathrm{II}}^{u}=\mathbf{0 , 6 0 3} \\
h_{\mathrm{I}}^{o}: g_{\mathrm{II}}^{u}=\mathbf{0 , 5 6 6} \\
g_{\mathrm{I}}^{o}: h_{\mathrm{II}}^{u}=\mathbf{0 , 5 8 5}\end{array}$}} \\
\hline $\begin{array}{l}49,6 \\
50,0\end{array}$ & $\begin{array}{l}19,0 \\
19,2\end{array}$ & 29 & 51,5 & 0,562 & & & & & \\
\hline $\begin{array}{l}49,9 \\
50,1\end{array}$ & $\begin{array}{l}19,5 \\
19,5\end{array}$ & 30 & 52,5 & 0,572 & & & & & \\
\hline
\end{tabular}

Zur Berechnung von $h_{\mathrm{I}}^{u} / h_{\mathrm{I}}^{o}$ sowie $g_{\mathrm{I}}^{u} / g_{\mathrm{I}}^{o}$ sind nicht die Werte der einzelnen dieser Größen aus den verschiedenen Tabellen miteinander zu kombinieren, sondern, um die Schwankungen des Lichtes zu eliminieren, die am Schluß der Tabellen angegebenen Verhältniszahlen. Es ergibt sich so, wenn die beiden Werte $g_{\mathrm{I}}^{o} / h_{\mathrm{II}}^{u}$ der Tabelle 2 noch zu gemeinsamem Mittel vereinigt werden:

$$
\left.\begin{array}{l}
h_{\mathrm{I}}^{u}: h_{\mathrm{I}}^{o}=\frac{0,727}{0,566}=1,284 \\
g_{\mathrm{I}}^{u}: g_{\mathrm{I}}^{o}=\frac{0,752}{0,594}=1,266
\end{array}\right\} \text { Mittel }: 1,275 .
$$


Um hieraus das Verbältnis der lichtelektrischen Strahlungsintensitäten $J_{40^{\circ}}$ und $J_{\tilde{0}^{\circ}} \mathbf{z u}$ gewinnen, ist $\mathrm{zu}$ berücksichtigen, daß der mittlere Inzidenzwinkel der Strablung, da die Platten vertikal hingen, bei der unteren Platte $40^{\circ}$, bei der oberen $5^{0}$ betrug, im übrigen die Intensitäten den Entladungszeiten umgekehrt proportional sind, so $\mathrm{daB}$

$$
\frac{J_{40} \cdot \cos 40}{J_{5}^{-} \cdot \cos 5}=\frac{1}{1,275},
$$

woraus folgt

$$
\boldsymbol{J}_{40^{\circ}}=1,02 \boldsymbol{J}_{50}
$$

Unter Berücksichtigung der einige Prozente betragenden Fehlergrenze heiBt dies, $\mathrm{daB}$ in zwei Richtungen, in denen die Intensitäten der sichtbaren Strahlung etwa im Verhältnis 5:1 stehen, die der lichtelektrischen Strahlung keinen nachweisbaren Unterschied aufweisen. Da die sichtbare Strahlung zu etwa \%/10 oder mehr vom Krater ausgeht, folgt, daB die lichtelektrische jedenfalls nur in sehr geringem Betrag vom Krater her kommt.

Nebenbei möge darauf hingewiesen werden, daB die Methode der Vertauschung der Platten, wie sie z. B. bei der obigen Anordnung verwendet ist, ermöglicht sowohl die Empfindlichkeit der Platten unabhängig von den Schwankungen des Lichtes, als auch die Konstanten der Zellen unabhängig von den speziellen Platten miteinander zu vergleichen. Seien $g, h$ die Ladungszeiten für den Fall, dab die Platten in einer Normalzelle, deren Empfindlichkeit 1 gesetzt wird, stecken, während die darauf fallende Strahlung einen bestimmten Wert $J$ hat, seien ferner $c_{\mathrm{I}}, c_{\text {II }}$ Apparatkonstanten, und zwar die Ladungszeiten, welche sich mit den betreffenden Apparaten ergeben, wenn diese eine Platte, die in der Normalzelle bei der Strahlungsintensität $J$ die Ladungsdauer 1 ergiebt, enthalten, dann ist $g_{\mathrm{I}}=g c_{1} ; g_{\mathrm{II}}=g c_{\mathrm{II}} ; h_{\mathrm{I}}=h c_{\mathrm{I}} ; h_{\mathrm{II}}=h c_{\mathrm{II}}{ }^{\circ} \quad$ Bei den Versuchen wird $g_{\mathrm{II}}$ und $h_{\mathrm{I}}$ gleichzeitig ermittelt, ebenso $g_{1 \mathrm{I}}$ und $h_{\mathrm{r}}$, so daB die Verhältnisse $g_{1} / h_{\mathrm{II}}$ und $h_{\mathrm{r}} / g_{\mathrm{II}}$ unabhängig von Schwankungen der Lichtstärke sind. Aus den obigen Gleichungen ergibt sich nun:

$$
\frac{g}{h}=\sqrt{\frac{g_{\mathrm{I}}}{h_{\mathrm{II}}} \cdot \frac{g_{\mathrm{II}}}{h_{\mathrm{I}}}} \text { und } \frac{c_{\mathrm{I}}}{h_{\mathrm{II}}}=\sqrt{\frac{g_{\mathrm{I}}}{h_{\mathrm{II}}} \frac{h_{\mathrm{I}}}{g_{\mathrm{II}}}} \text {. }
$$


Auf diesem Weg liefern die Tabellen 1 und $2 g / h=1,017$ bez. 1,024. Die Übereinstimmung beider Werte bestätigt die Brauchbarkeit der Beobachtungen. Für $c_{1} / c_{\mathrm{nI}}$ ergibt Tabelle 1 den Wert 0,740. Wenn es erforderlich ist, lassen sich die Konstanten natürlich durch Zuschalten von Kapazität oder geeignete Wahl der Endpotentiale abgleichen.

Bei einer 4 Monate später vorgenommenen Wiederholung der vorigen Versuche wurde der schräge Einfall der Strahlen auf die untere Platte vermieden, letztere nahezu senkrecht zu denselben orientiert. Der noch bleibende Inzidenzwinkel betrug 5,50. Die Platte der oberen Zelle stand normal zur Strahlung. Die Beobachtung der Ladungszeiten lieferte folgende Werte (Sekunden):

Tabelle 3.

\begin{tabular}{|c|c|c|c|c|c|}
\hline$y_{\mathrm{II}}^{0}$ & $h_{\mathrm{I}}^{n 6}$ & $g_{\mathrm{II}}^{0} / h_{\mathrm{I}}^{u}$ & $h_{1 \mathrm{I}}^{o}$ & $g_{1}^{u}$ & $h_{11}^{o} / g_{\mathrm{I}}^{u}$ \\
\hline 127 & 123 & 1,033 & 129 & 120 & 1,075 \\
\hline 121 & $1 ! 9$ & 1,017 & 125 & 118 & 1,059 \\
\hline \multicolumn{5}{|c|}{ Mittel: 1,025} & 1,067 \\
\hline
\end{tabular}

Hieraus folgt:

$$
c_{\mathrm{II}}^{0} / c_{\mathrm{I}}^{u}=\sqrt{1,025 \cdot 1,067}=1,046 .
$$

Das Verbältnis $c_{\mathrm{II}}^{\circ} / c_{\mathrm{I}}^{0}$ ergab sich aus zwei Versuchsreihen, welche einige Tage vor- bez. nachher stattfanden, zu 1,045 bez. 1,071 ; Mittel $1,058,{ }^{1}$ ) so da $B$

und da

$$
c_{\mathrm{I}}^{u} / c_{\mathrm{I}}^{o}=1,058 / 1,046=1,012,
$$

so folgt

$$
J_{0} / J_{40} \cos 5,5^{0}=c_{\mathrm{I}}^{u} / c_{\mathrm{I}}^{0},
$$

$$
\boldsymbol{J}_{40^{\circ}}=\mathbf{0 , 9 9} \boldsymbol{J}_{0^{\circ}},
$$

welches Ergebnis mit dem der ersten Beobachtungsreihe bestens übereinstimmt.

B) Strahlung über und unter die Kraterebene.

Das Vorige wurde nun durch einige Versuche ergänzt. Bei dem ersten derselben lag zunächst der obere Rand der

1) Die Zellen waren inzwischen abgeglichen worden. 
bestrahlten Platten noch ein wenig unterhalb der Kraterebene, so daB von allen Punkten der wie immer $90 \mathrm{~cm}$ entfernten Platten aus noch ein kleiner Teil des Kraters sichtbar blieb. Zelle II behielt diese Stellung dauernd bei, während I für einen weiteren Versuch $12 \mathrm{~cm}$ höher zu stehen kam, so daB der Mittelpunkt der bestrahlten Platte $4,4 \mathrm{~cm}$ oberhalb der Horizontalebene durch den Krater lag und ihr unterer Rand nur noch $1,8 \mathrm{~cm}$ unter die letztere hinabreichte. Tabelle 4 gibt die erhaltenen Ladungszeiten und $z$ war bedeutet $g_{1}$ diejenige bei der tieferen, $g_{\mathrm{I}}^{\prime}$ die bei der höheren Lage von $\mathrm{I}$, $h_{\mathrm{II}}$ die von II.

Tabelle 4.

\begin{tabular}{cl|ll|ll}
\hline$h_{\mathrm{II}}$ & $g_{\mathrm{I}}{ }^{\prime}$ & $h_{\mathrm{II}}$ & $g_{\mathrm{I}}$ & $h_{\mathrm{II}}$ & $g_{\mathrm{I}}{ }^{\prime}$ \\
\hline \hline 42 & 25,5 & 42 & 25 & 42 & 25 \\
42 & 25,5 & 41,5 & 25 & 42 & 25,5 \\
41 & 25 & 42 & 25 & 42 & 25,5 \\
42,5 & 26 & - & - & - & -
\end{tabular}

Hieraus ergibt sich $g_{\mathrm{I}}^{\prime} / h_{\mathrm{II}}=0,609$ bez. 0,601 , im Mittel 0,605 , und $g_{\mathrm{I}} / h_{\mathrm{II}}=0,597$, woraus folgt

$$
g_{\mathrm{I}}^{\prime}=1,01 g_{\mathrm{I}} \text {. }
$$

Es zeigt sich kein merkbarer Unterschied der Strahlung für die beiden Lagen, woraus mit noch gröBerer Sicherheit hervorgeht, daB rom Krater kein merkbarer Teil der lichtelektrischen Strahlung kommt. Was die Zahlenwerte der Tabelle 4 betrifft, so muß darauf hingewiesen werden, daß eine so große Übereinstimmung, wie sie dieselben zeigen, nur ausnahmsweise zu erzielen war; meist variierte die Stärke der Strahlung viel beträchtlicher.

Bei einer 4 Monate späteren Wiederholung der letzten Versuche wurde mit einer bis auf einen schmalen Horizontalspalt abgeblendeten Linse, welche in der Nähe des Ortes aufgestellt war, an welchen die Zellen kommen sollten, ein Bild des Lichtbogens entworfen. Durch vertikales Verschieben der Linse, bis gerade eben der letzte Saum des Kraters auf dem Bild verschwand, lieb sich dann die Stelle aufsuchen, welche eben nicht mehr von Kraterstrahlen getroffen werden konnte. Die Zellen erhielten dann dicht vor der Linse ihre Aufstellung 
und zwar Zelle I stets mit ihrem oberen, Zelle II erst mit ihrem oberen, dann mit ihrem unteren Rand gerade vor dem Linsenspalt. Auf diese Weise lieb sich schärfer bewirken, $\mathrm{daB}$ in der letzten Lage Zelle II von Kraterstrahlen gerade eben nicht mehr getroffen werden konnte. Aus den beobachteten Ladungszeiten ergab sich:

$$
h_{\mathrm{II}}^{\prime}=1,00 h_{\mathrm{II}}
$$

in bester Übereinstimmung mit dem vorigen Resultat.

Als dann die Platte II noch etwa $1 \mathrm{~cm}$ höher gerückt wurde, zeigte sich eine Abnabme der Strahlung um 6 Proz. Eine Abnahme nach oben hin wird zum Teil dadurch bedingt, $\mathrm{daB}$ das verhältnismäBig dicke Ende der oberen, positiven Kohle mit der Erhebung der Strahlrichtung über die Horizontale beträchtliche Teile des Bogens abblendet. Bei Neigung unter die Horizontale ist eine solche Abblendung, welche dort durch die negative Kohle bewirkt werden müBte, wegen der Zuspitzung der letzteren, auch wegen ihrer kleineren Dicke nur gering, wobei noch hinzu kommt, daB der Bogen selbst gegen die negative Kohle hin sehr dünn wird. So erhalten Orte in Richtungen, welche selbst $40^{\circ}$ unter die Horizontale geneigt sind, Strahlen noch fast von allen Teilen des Bogens.

Diese Erklärung reicht indessen quantitativ nicht aus. Der quantitativ beträchtliche Unterschied zwischen der Abnahme der Strahlung nach oben und der Abnabme nach unten hin macht vielmehr wahrscheinlich, daß die Strahlung des Bogens von der positiven nach der negativen Kohle hin kleiner wird. Wenn also die in diesem Paragraphen besprochenen Versuche den Nachweis liefern, daß die lichtelektrische Strahlung nicht vom Krater her kommt, so bleibt noch zu untersuchen, wie dieselbe auf den Bogen verteilt ist. Über diesbezügliche Versuche wird im $\S 4$ berichtet werden.

\$ 3. Grundzüge eines Strahlungsgegetzes des Lichtbogens.

Nachdem die lichtelektrische Strahlung als spezifische Bogenstrahlung erwiesen ist, erheischt die Untersuchung der Abhängigkeit der Strahlungsintensität von Spannung und Strom, welche sich eventuell als Strahlungsgesetz des Bogens ansprechen lassen wird, besonderes Interesse. Die Kenntnis 
dieser Beziehung würde auch bei lichtelektrischen Arbeiten die Reduktion der Lichtstärken auf bestimmten Strom und Spannung gestatten, sowie Aussicht für Aufklärungen über die Vorgänge im Lichtbogen bieten.

Ganz im Gegensatz zur sichtbaren Strahlung, welche, abgesehen von kurzen Bögen, bei denen die Beschattung durch die Kohlen eine zu bedeutende Rolle spielt, von der Spannung nur in geringem $\mathrm{MaB}$ abhängig ist, tritt bei der lichtelektrischen Strahlung schon nach meinen früheren gelegentlichen Erfahrungen die Abhängigkeit von der Elektroden- oder besser von der Bogenspannung stark hervor.

a) Abhangigkeit der Strahlungsintensitat von der Spannung.

Zunächst wurde daher bei möglichster Konstanthaltung der Stromstärke die Spannung variiert. Dies lieB sich mit einer Hauptstromlampe ausführen, und zwar kam eine der in physikalischen Instituten überall verbreiteten, älteren HefnerAlten eckschen Kontaktlampen zur Anwendung. Aufstellen der letzteren in Vaselinöl, so daB die Luftdämpfungsflügel ihre Bewegung in dieser Flüssigkeit ausführen mußten, und Anbringen eines genügenden Kompensationsgewichtes für den oberen Kohlenhalter verfeinerten die Regulierung so, daB sie derjenigen der sonst benutzten, oben erwähnten Piette-KriłikLampe fast gleichkam. Die Variationen der Stromstärke hielten sich bei $20 \mathrm{Amp}$. Strom innerhalb eines Ampers.

Zuerst wurde die Strahlungsintensität bei Spannungen in der unmittelbaren Nähe der für Beleucbtungszwecke bei etwa 48 Volt liegenden Gebrauchsklemmspannung der Lampe untersucht, weil gerade in diesem Intervall die Zunahme der Strahlung mit der Spannung ganz besonders rasch zu sein schien. Die Regulierung der Lampe war dabei auf 20,5 Amp. eingestellt. Da es einiger Zeit bedurfte, bis die Kohlen genügend einbrannten und die Strahlung verschiedener Kohlen selbst der nämlichen Fabrikation nicht unbeträchtlich roneinander abweichen, so gelangte jede Spannung gesondert zur Untersuchung, indem die Beobachtung der Ladungszeit bei derselben zwischen zwei Beobachtungssätze bei 48 Volt gelagert wurde. Auf diese Weise war man unabhängig vom Kohlenwechsel und konnte die Entladungszeiten bei den einzelnen Spannungen 
durch Reduktion auf den für 48 Volt erhaltenen Mittelwert miteinander vergleichbar machen.

Die folgende Tabelle 5 gibt zunächst als Beispiel für den Verlauf der Beobachtungen im einzelnen zwei vollständige Beobachtungssätze. $P$ ist die Spannung in Volt, $\tau_{1}$ und $\tau_{2}$ sind die am Elektroskop beobachteten Ladungszeiten zweier lichtelektrischer Zellen; die Werte von $P$ bilden die Umkehrpunkte des Spannungsmessers während der Bestimmung von $\tau$. Im Beobachtungssatz für $P=46,0$ Volt traten bei der langen Dauer von $\tau_{2}$ mehrere Umkehrpunkte ein, wodurch sich die Striche in der Tabelle erklären. In die Ermittelung der Werte teilten sich zwei Beobachter.

Tabelle 5 .

\begin{tabular}{|c|c|c|c|c|c|c|c|c|c|c|c|}
\hline \multicolumn{2}{|c|}{$P$} & $\tau_{1}$ & $\tau_{2}$ & \multicolumn{2}{|c|}{$P$} & $\tau_{1}$ & $\tau_{2}$ & \multicolumn{2}{|c|}{$P$} & \multirow{2}{*}{$\frac{\tau_{1}}{\bar{~}}$} & \multirow{2}{*}{$\frac{\boldsymbol{\tau}_{2}}{40}$} \\
\hline 48,3 & 47,9 & 17,5 & 36 & 45,8 & 46,4 & 31 & 73,5 & 48,2 & 47,8 & & \\
\hline 48,4 & 47,9 & 17 & 36,5 & 45,5 & 46,2 & - & - & 48,0 & 48,2 & 18 & 40 \\
\hline 47,9 & 48,3 & 17,5 & 39,5 & 45,7 & 46,2 & 31 & 72 & 48,1 & 48,3 & 17,5 & 39,5 \\
\hline 47,8 & 48,2 & 17,5 & 39,5 & 46,2 & 45,8 & 30 & 70 & 47,8 & 48,5 & 17,5 & 39,5 \\
\hline 47,8 & 48,2 & 17,5 & 40 & 46,1 & 45,8 & $\dot{-}$ & - & 47,8 & 48,2 & 18 & 40,5 \\
\hline 47,8 & 48,3 & 17,5 & 40 & 45,6 & 46,2 & 31,5 & 72,5 & - & - & - & - \\
\hline- & - & - & - & 45,7 & 46,2 & - & - & - & - & $=$ & - \\
\hline Mittel & $\mathbf{4 8 , 1}$ & 17,4 & 39,2 & 46 & & $\mathbf{3 0 , 9}$ & 72,0 & 48 & & 17,9 & 39,9 \\
\hline 48,1 & 47,7 & 20 & 41 & 48,9 & 49,3 & 17 & 35 & 47,9 & 48,2 & 19 & 41 \\
\hline 47,9 & 48,5 & 18 & 37 & 48,7 & 49,2 & 17 & 37 & 47,8 & 48,1 & 19,5 & 42 \\
\hline 47,9 & 48,4 & 18 & 37 & 49,2 & 48,7 & 17,5 & 37 & 47,9 & 48,2 & 20 & 42 \\
\hline 47,9 & 48,3 & 18 & 38 & 48,7 & 49,2 & 17 & 37 & - & - & - & - \\
\hline 48,0 & 48,6 & 17,5 & 37 & - & - & - & 一 & - & - & - & - \\
\hline 48,2 & 47,7 & 19 & 40 & - & - & - & - & - & - & - & - \\
\hline 47,7 & 48,1 & 19 & 42 & - & $=$ & - & - & - & - & - & - \\
\hline Mittel & 48,1 & 18,5 & 38,9 & & & 17,1 & 36,5 & & & 19,5 & 41,7 \\
\hline
\end{tabular}

Diese Tabelle liefert eine Übersicht über die erreichbare Genauigkeit. Die Anordnung gestattete die Zeiten etwa auf $1 / 2$ Sek. genau festzustellen: eine größere Genauigkeit hätte wegen der unvermeidlichen Intensitätsschwankungen keinen Zweck gehabt. Andererseits zeigt die Tabelle indes auch, daB die bei aller Sorgfalt im Zentrieren der Kohlen mit einer gut in Stand gesetzten Lampe und besten Kohlen erzielbare Konstanz nicht unterschätzt zu werden braucht. 
Die folgende Tabelle 6 enthält eine Zusammenstellung der SchluBresultate der nach dem Beispiel der Tabelle 5 ausgeführten Beobachtungssätze. Dabei wurden die bei den verschiedenen Spannungen gefundenen Werte für $\tau$ auf gleiche Verhältnisse reduziert, indem man sie mit dem Verhältnis des Mittels aller für 48 Volt gefundenen $\tau$ zu dem zum jeweiligen Satz gehörigen Wert für 48 Volt multiplizierte.

\begin{tabular}{ccccccc}
\multicolumn{7}{c}{ Tabe 1le 6.} \\
$P$ & 50,8 & 50,0 & 49,0 & 48,0 & 47,0 & 46,0 \\
$\tau_{1}$ & 12,5 & 13,5 & 16,1 & 17,9 & 20,5 & 31,3 \\
$\tau_{2}$ & 27,0 & 29,5 & 35,1 & 38,8 & 45,4 & 70,6
\end{tabular}

Unter 46 Volt mit der Spannung herabzugehen hatte keinen Zweck, da schon bei 45 Volt die Strahlung sehr inkonstant wird, was mit dem Kleinwerden des Bogens zusammenhängt. Vor der in Tabelle 6 in ihren Resultaten angegebenen Beobachtungsreihe waren schon 2 andere, weniger umfangreiche ausgeführt worden (Platte 2 war damals noch empfindlicher wie bei Tabelle 6); das Ergebnis derselben, und zwar der Einzelwerte der beiden Reihen zum Mittel vereinigt, enthält Tabelle 7 .

\begin{tabular}{|c|c|c|c|c|}
\hline \multicolumn{5}{|c|}{$\begin{array}{c}\text { Tabelle } 7 \\
i=20,5 \text { Amp. }\end{array}$} \\
\hline 50,0 & 49,0 & 47,9 & 47,0 & 46,0 \\
\hline 13,9 & 15,9 & 19,3 & 21,8 & 32,0 \\
\hline 26,2 & 28,5 & 35,4 & 41,2 & 57,4 \\
\hline
\end{tabular}

Aus den Werten der beiden letzten Tabellen ist $z u$ schlieBen, daB die Strahlungsintensität jedenfalls nicht mit der Gesamtspannung und damit mit der gesamten zugeführten Energie in einfacher Beziehung steht. Der in $\S 2$ geführte Nachweis, daB die Strahlung vom Bogen ausgeht, weist darauf hin, zu untersuchen, ob ihre Intensität mit dem auf den Bogen entfallenden Teil der Spannung, den wir mit $p-p_{0}$ bezeichnen wollen, einen einfachen Zusammenhang besitzt; deshalb waren auch für die gerade besprochenen ersten Versuchsreihen Spannungen in der Nähe ron $p_{0}$ gewählt worden. Proportional 
mit $p-p_{0}$, d. h. mit der dem Bogen zugeführten Energie, ist die Intensität, wie die Überprufung der Tabellenwerte (6 und 7) ergibt, keinesfalls, indes geht sie sehr nahezu proportional mit der Wurzel aus $p-p_{0}$. Aus dem Mittel der beiden in Tabelle 6 und 7 gegebenen Reihen fand sich, daß, wenn man $p_{0}=44,7$ nimmt, die $\sqrt{p-p_{0}}$ als Funktion von $1 / \tau$ graphisch dargestellt, sehr nahezu eine Gerade liefert.

Vor weiterer Verfolgung der Möglichkeit dieser Darstellung gelangten nun Versuche mit stärkerer Variation der Spannung zur Ausführung. Die Möglichkeit der Ausdehnung des Intervalles beschränkte sich etwa anf das Gebiet von 46--70 Volt: an der oberen Grenze ist es schon äuBerst schwierig, den Bogen während einer Zeit, wie sie das richtige Einbrennen der Kohlen erfordert, vor dem Wandern zu bewahren, an der unteren Grenze fangen bereits die UnregelmäBigkeiten der Kohlenränder an, den Öffnungswinkel des klein gewordenen Bogens merklich zu beeinflussen. Das Ergebnis der erwähnten Versuche enthält Tabelle 8 , in welcher die Ladungszeiten wegen geänderter Platten mit $\tau_{1}{ }^{\prime}$ und $\tau_{2}{ }^{\prime}$ bezeichnet sind.

Tabelle 8.

$\begin{array}{llllll}P & 51,0 & 54,6 & 60,7 & 65,7 & 52,5 \\ i & 20,1 & 20,8 & 20,1 & 21,3 & 20,4 \\ \tau_{1}{ }^{\prime} & 43 & 30 & 23 & 19 & 35 \\ \tau_{2}{ }^{\prime} & 69 & 47,5 & 36,5 & 31 & 56\end{array}$

Um auch für diese Versuche mit höheren Spannungen, bei welchen übrigens, da die Siemenslampe damals anderweitige Verwendung hatte, die Schuckertlampe gebraucht wurde, ein Urteil über den Verlauf im einzelnen zu ermöglichen, sind in der Tabelle 9 die Einzelablesungen bei der höchsten der benutzten Spannungen, bei welcher die Konstanthaltung am schwierigsten war, aufgef ührt. Die Stromstärken $i$ mußten hier mit notiert werden, weil die Lampe als Differentiallampe nicht auf bestimmtem Strom reguliert. Hinsichtlich der Spannungsverluste in den Lampenteilen unterschieden sich die beiden Lampen nicht wesentlich. ${ }^{\text {) }}$

1) Eine schärfere Berücksichtigung der Spannungsverluste behalte ich mir für weitere Beobachtungen vor. 
Tabelle 9.

\begin{tabular}{|c|c|c|c|c|c|c|c|}
\hline \multicolumn{3}{|c|}{$P$} & \multicolumn{3}{|c|}{$i$} & $\tau_{1}^{\prime}$ & $\boldsymbol{\tau}_{2}{ }^{\prime}$ \\
\hline 64,5 & 65,2 & 66,0 & 22,5 & 21,0 & 21,5 & 19 & 31 \\
\hline 66,0 & 66,2 & 66,0 & 21,5 & 21,6 & 22,0 & 18 & 30 \\
\hline 66,0 & 65,8 & 64,8 & 21,0 & 21,5 & 21,0 & 18 & 29,5 \\
\hline 66,1 & 66,0 & 66,2 & 20,0 & 20,5 & 21,0 & 20 & 34 \\
\hline
\end{tabular}

Für die Beobachtungen der Tabelle 8 sowohl, als auch für die zu Mittelwerten vereinigten der Tabellen 6 und 7 gibt die Fig. 1 eine graphische Darstellung, bei welcher zu den $\tau$ als Abszissen die Werte $1 / \sqrt{p-44,7}$ die Ordinaten bilden. Diese Darstellung zeigt, daB zwischen letzterem Ausdruck und der der reziproken Strahlungsintensität proportionalen Zeit $\tau$ eine lineare Beziehung besteht. Dabei laufen die Geraden nicht durch Null, es lassen sich aber, wie aus der Figur ersichtlich, die für die vier verschiedenen Platten gültigen Geraden unter Berücksichtigung der hier möglichen Genauigkeit durch einen Punkt führen, für dessen Koordinaten aus der Figur unter Hinzunahme einiger Hilfsrechnungen $\tau=0$ und $1 / \sqrt{p-44,7}=0,058$ erhalten wurde. Bezeichnen daher $\tau_{10}, \tau_{20}$ etc., allgemein $\tau_{0}$, Plattenkonstanten, so ist

$$
\frac{r}{\tau_{0}}+0,058=\frac{1}{\sqrt{p-}}-\overline{44,7} \text { (vgl. Anm. p. 59). }
$$

Die beiden numerischen Konstanten sind für dic verschiedenen Platten dieselben, die $\tau_{0}$ sind von der Spannung unabhängig, natürlich aber der Empfindlichkeit der gewählten Versuchsanordnung umgekehrt proportional und von der Stromstärke abhängig.

B) Abhängigkeit von der Stromstärke.

Vorbehältlich der Überprüfung durch weitere Versuche, vgl. unten, stellt die vorige Formel die Abhängigkeit der Strahlung von der Spannung genügend dar, so daß zur Untersuchung der Abhängigkeit von der Stromstärke übergegangen werden kann. Bei den diesbezüglichen Versuchen wurde, weil keine Nebenschlußlampe, die auf konstante Spannung reguliert hätte, zur Verfügung stand, die vorher benutzte Differentiallampe genommen. Da sie auf konstanten Widerstand reguliert, 
Strahlung des Lichtbogens.

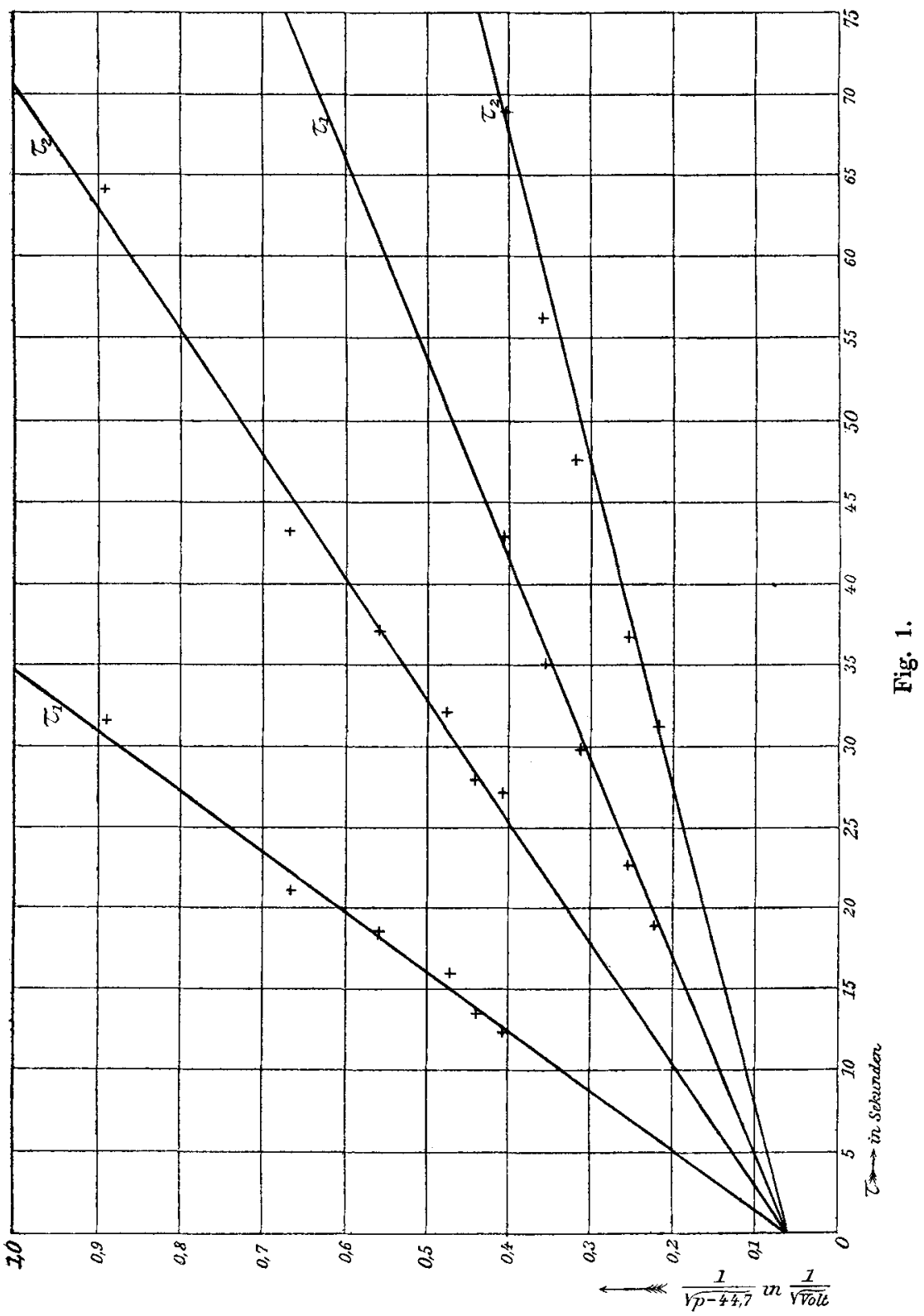


erhielt ihre NebenschluBspule nach der dazu erforderlichen Lösung innerer Verbindungen einen separaten Vorschaltwiderstand, so daB man die Spannung unabhängig von den Stromvariationen konstant halten konnte. Einen derartigen Vorschaltwiderstand, der übrigens auch bei einem Teil der vorigen Versuche schon zur Anwendung kam, habe ich auf die Dauer auch bei anderen lichteleltrischen Versuchen für praktisch gefunden. Die NebenschluBspule der Lampe vermehrt ja, wenn die Lampe wärmer wird, ihren Widerstand, weshalb die Spannung, auf welche die Lampe reguliert, wächst, da die Empfindlichkeit der Hauptstromspule dieselbe bleibt. Infolgedessen steigt die von der Spannung so sehr abhängige lichtelektrische Strahlungsintensität mit der Zeit an. Dem läBt sich durch Einschaltung eines Widerstandes vor die NebenschluBspule, den man mit dem weiteren Brennen der Lampe entsprechend verkleinert, abhelfen. Überdies wird dadurch ein schnelleres Einbrennen der Lampe ermöglicht. Es ist ferner zweckmäßig, die Nebenschlußspule bereits einige Zeit vor dem Anbrennen der Lampe unter Strom zu setzen, damit sie sich erwärmt, und so ihren Endwiderstand bei Beginn der Versuche schon möglichst erreicht hat. Den erwähnten Übelstand dadurch zu beseitigen, daß die Nebenschlußspule Konstantanwickelung oder dergl. erhält, läBt sich nach Anfrage bei der Fabrik leider nicht bewerkstelligen, weil der Raum für die erforderliche Ampèrewindungszahl fehlt.

Die Anordnung der Versuche zur Ermittelung der Abhängigkeit der Strahlung von der Stromstärke schlob sich der p. 48 angegebenen an. Die Spannung wurde in nächster Nähe von 55 Volt $(54,7-55,5)$ gehalten und die Versuche zunächst mit Hilfe der Formel (1) p. 52 auf 55 Volt, sodann gemäB p. 50, da zwei verschiedene Kohlen nötig gewesen waren, auf gleiche Kohle reduziert. So ergaben sich die Werte der folgenden Tabelle.

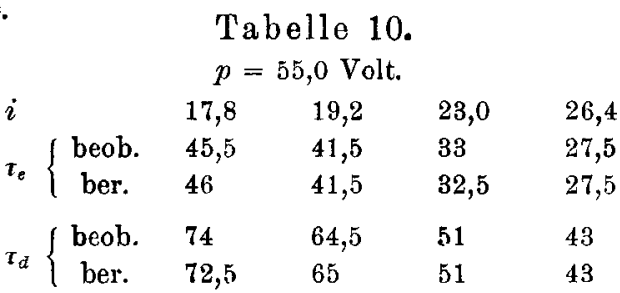


Die Versuche zeigen, daß bezüglich der Abhängigkeit von der Stromstärke die lichtelektrische Strahlung sich ganz analog verhält wie die sichtbare. Die berechneten Werte der Tabelle sind einfach mit Hilfe der Formel:

$$
\tau\left(i-i_{0}\right)=c, \text { wo } i_{0}=5,3
$$

berechnet, indem das Mittel der aus den einzelnen Versuchen hervorgehenden Werte von $c$ durch die einzelnen Werte von $(i-5,3)$ dividiert wurde. Die von Blondel gegebene Kurve für die Abhängigkeit der sichtbaren Lichtstärke von der Stromstärke ${ }^{1}$ ) bei konstanter Spannung fällt bis zu $10 \mathrm{Amp}$. herab ebenfalls mit einer Geraden sehr nahe zusammen, die auch auf einen entsprechenden Punkt der Abszisse, etwa 6 Amp., hinzielt. Unter $10 \mathrm{Amp}$. herab und namentlich bis zu kleinen Stromstärken von wenigen Ampèren hin reicht diese einfache Darstellung keineswegs aus. Auf diese kleinen Stromstärken kam es mir aber bei dieser Untersuchung weniger an, da dort die lichtelektrische Strahlung sehr schwach ist, und im Unterschied zu den größeren Stromstärken die Verbältnisse sich zu verwickeln scheinen. Auf ersteres deutet ja die obige Formel hin: wenn auch die Strahlung bei sehr kleinen Stromstärken nicht auf Null herabgeht, so schwächt sie sich doch auBerordentlich ab. Dies bestätigen Versuche, welche mit einer Siemensschen Liliputlampe (ohne Glocke) ausgeführt wurden. Bei diesen ergab sich:

$\begin{array}{cccc}P & \dot{i} & \tau_{e} & \tau_{j} \\ 71 & 2,47 & 1390 & 1240 \\ 49,5 & 20,2 & 57,5 & 47,8\end{array}$

In der unteren Reihe sind unmittelbar vorher unter sonst gleichen Verhältnissen mit einer anderen Lampe angestellte Beobachtungen angegeben. Die Spannung der Liliputlampe schwankte unaufhörlich zwischen 67 und 75 Volt, die Stromstärke zwischen 2,34 und 2,65 Amp. Während der Zeitbeobachtung fanden 100 Ablesungen der Spannung und 40 der Stromstärke statt. Unter Berücksichtigung der Mängel der Isolation würden die Zeiten der oberen Reihe noch etwa

1) Vgl. H. Ayrton, The El. Are., 1903. p. 367 bez. A. Blondel, Eclairage électrique 10. p. 498. 1897. 
10 Proz. größer. Zieht man, was nach den weiter unten folgenden Versuchen als zulässig erachtet werden muß, die Formel(1) zur Berechnung der Zeiten $\tau$ heran, welche mit der Liliputlampe erhalten würden, wenn die Strahlung der Stromstärke direkt proportional wäre, so sollten dieselben 3,4 mal so groB werden wie bei 49,5 Volt und 20,2 Amp. Tatsächlich sind sie 24-26 mal so groB. Die Abnahme der Wirkung ist also etwa 7,5 mal so stark, als es sein sollte, wenn in der Formel (2) das Glied $i_{0}$ wegkäme. Nas Einfügen dieses $i_{0}$, welches besagt, daB unter 5,3 Amp. keine im Vergleich zu der bei etwa $20 \mathrm{Amp}$. erhebliche Wirkung mehr vorhanden ist, rechtfertigt sich also. Zur Berechnung des vorläufigen Zahlenwertes von $i_{0}$ sind außer den Versuchen der Tab. 10 auch viele der anderen Versuche noch herangezogen worden.

Wenn man annimmt, daB die Bogenstrahlung von Teilchen ausgeht, welche ursprünglich im Krater im Zusammenhang mit der dort herrschenden hohen Temperatur gebildet worden sind, so findet die nahe gleichlaufende Abhängigkeit der sichtbaren und der lichtelektrischen Strahlung von der Stromstärke ihre Erklärung.

y) Gleichzeitige Variation von Stromstärke und Spannung.

Drei weitere Versuchsreihen sollten sowohl zur ferneren Prüfung der Formeln dienen, als auch zur Feststellung, ob Strom und Spannung genügend unabhängig wirken, um Vereinigung der beiden Formeln zu gestatten in:

(3) $\quad \tau_{p, i}=\frac{T_{0}}{i-5,3}\left[\frac{1}{\sqrt{p-41,7}}-\mathbf{0 , 0 5 8}\right]$ (vgl. Anm. p. 59).

wo $T_{0}$ eine Konstante der Versuchsanordnung und jeweiligen Platte.

Zwei dieser Versuchsreihen fanden mit horizontaler, eine mit um $40^{\circ}$ unter den Horizont geneigter Strahlung statt. Strom und Spannung wurden gleichzeitig variiert. In der die Ergebnisse enthaltenden Tab. 11 folgten die berechneten Werte der $\tau$ aus den unter den Reihen stehenden $T_{0}$, welche das Mittel der aus den Einzelbeobachtungen gewonnenen Werte dieser GröBe sind, und Formel (3). In den beiden ersten Versuchsreihen kamen zwei, in der letzten nur eine Zelle zur Exposition. 
Tabelle 11.

\begin{tabular}{|c|c|c|c|c|c|}
\hline \multirow{2}{*}{$P$} & \multirow{2}{*}{$\imath$} & \multicolumn{2}{|c|}{$\tau^{\prime}$} & \multicolumn{2}{|c|}{$\boldsymbol{x}^{\prime \prime}$} \\
\hline & & beob. & ber. & beob. & ber. \\
\hline 65,8 & 26,2 & 13 & 12,5 & 20 & 20 \\
\hline 60,4 & 23,7 & 17 & 17 & 27,5 & 27,5 \\
\hline 55,5 & 21,9 & 23 & 24 & 38,5 & 38,5 \\
\hline 50,8 & 21,0 & 34 & 35,5 & 55 & 57 \\
\hline \multirow[t]{2}{*}{47,6} & 18,2 & $68, \overline{0}$ & 67 & 109 & 107 \\
\hline & & \multicolumn{2}{|c|}{$T_{0}^{\prime}=1610$} & \multicolumn{2}{|c|}{$T_{0}^{\prime \prime}=2580$} \\
\hline 70,4 & 27,7 & 10 & 11 & 16,5 & 17,5 \\
\hline 65,8 & 26,7 & 13 & 13 & 21,5 & 21 \\
\hline 52,5 & 20,4 & 35 & 34,5 & 56 & 56 \\
\hline \multirow[t]{2}{*}{51,0} & 20,1 & 43 & 40 & 69 & 65,5 \\
\hline & & \multicolumn{2}{|c|}{$T_{10}^{\prime}=1730$} & \multicolumn{2}{|c|}{$T_{10}^{\prime \prime}=2820$} \\
\hline 65,4 & 28,2 & 24 & 24,5 & & \\
\hline 66,5 & 22,5 & 32,5 & 32 & & \\
\hline 47,8 & 19,2 & 131 & 130 & & \\
\hline \multirow[t]{2}{*}{47,9} & 15,3 & 175 & 175 & & \\
\hline & & \multicolumn{2}{|c|}{$T_{0}=3500$} & & \\
\hline
\end{tabular}

Die Übereinstimmung der beobachteten mit den nach Formel (3) berechneten Werten ist so gut, wie sie in Anbetracht der erreichbaren Konstanz der Strahlung nur erwartet werden kann. Die Formel (3) stellt daher innerhalb des Intervalles von 46-70 Volt und 15-28 Amp. die lichtelektrische Strahlungsintensität in ihrer Abhängigkeit von Spannung und Strom vorläufig genügend dar. Eine Untersuchung darüber, ob die Formel auch bei noch größeren Stromstärken und Spannungen weiter anwendbar ist, führte bisher nicht zum Ziel, da die vorhandenen Mittel nicht ausreichten, den Bogen bei $40 \mathrm{Amp}$. und 80 Volt genügend konstant zu bekommen.

Das für das angegebene Intervall ermittelte Strahlungsgesetz hat inzwischen bei anderen lichtelektrischen Untersuchungen gute Dienste geleistet, indem es vielfach das zeitraubende Einbrennen des Bogens auf ganz bestimmten Strom und Spannung für solche Versuche, welche miteinander verglichen werden sollten, ersparte, da es die Möglichkeit der Reduktion der Strahlungsintensitäten aufeinander gewährt. 
Das Zustandekommen der Formel (3) könnte unter anderem unter Umständen durch folgende Betrachtung erläutert werden. Man kann annehmen, daB die lichtelektrische Strahlung von Teilchen ausgeht, welche den Bogen durchlaufen und während dessen wesentlich in konstanter Weise strahlen. Sie werden ursprünglich am Krater entstanden sein, worauf schon oben p. 56 hingewiesen worden ist. Da der Krater nach den vorliegenden Untersuchungen ${ }^{1}$ ) konstante, von Strom und Spannung unabhängige Temperatur besitzt, so liegt die gemachte Annahme konstanter Strahlung der Teilchen nahe. Von diesen Teilchen soll weiter angenommen werden, daß ihr Transport durch den Bogen mit dem Stromphänomen verknüpft ist, und sie infolgedessen vom Eintritt in den Bogen ab sowohl Beschleunigung als auch bestimmte lichtelektrische Strahlungsintensität erhalten. Die Anzahl der Teilchen würde $i$ proportional sein, wenn nicht schon die Kraterstrahlung derselben nicht proportional wäre (vgl. p. 55) und dadurch darauf hinwiese, daß die Ränder des Kraters durch die anliegenden Teile der positiven Kohle eine Abkühlung erfahren. Diesem Umstand läßt sich, wie erwähnt, in dem bei den vorliegenden Versuchen benutzten Intervall dadurch Rechnung tragen, daB man die sichtbare Strahlung dem Ausdruck $i-i_{0}$ proportional nimmt, was dann, gemäB dem im vorigen angenommenen $\mathrm{Zu}$ sammenhang der lichtelektrisch strahlenden Teilchen mit dem Krater, auch für die lichtelektrische Strahlung gilt, so daB dieselbe zunächst $i-i_{0}$ proportional zu setzen ist.

Sie ist ferner der Dauer $t$ des Aufenthaltes der Teilchen im Bogen proportional zu nehmen. Setzen wir voraus, daB die Teilchen mit der Geschwindigkeit Null in der Richtung des Bogens in diesen eintreten und im Bogengebiet die Beschleunigung $b$ erhalten, während sich eventuell dieser Bewegung nur der Beschleunigung proportionale Widerstände entgegensetzen, so ist, wenn noch $l$ die Bogenlänge bedeutet:

$$
t=c \sqrt{\frac{l}{b}} \text {. }
$$

Die mittlere Beschleunigung $b$ kann in erster Annäherung konstant gesetzt werden, da für das von uns benutzte Intervall

1) Die Literatur vgl. H. Ayrton, l. c. p. 347. 
die auf den Bogen entfallende Potentialdifferenz der Bogenlänge, soweit die Untersuchungen darüber reichen, sehr nahe proportional ist. Wir erhalten daher:

$$
t=k \sqrt{P-P_{0}} \text {, }
$$

wo $k$ eine Konstante ist, und für die Strahlungsintensität $S$ würde sich ergeben:

$$
S=C\left(i-i_{0}\right) \sqrt{P-\bar{P}_{0}}
$$

wo $C$ eine andere Konstante. Dieser Ausdruck fällt mit dem experimentell gefundenen sehr nahe zusammen. Er ließe sich damit ganz in Einklang bringen, wenn ins Auge gefaBt wird, $d a B$ die Teilchen im allgemeinen nicht gerade, sondern gekrümmte Bahnen durchlaufen und sich demgemä日 länger im Bogen aufhalten. Eine Korrektion hierfür in einfacher Form ergäbe sich unter Berücksichtigung dessen, daß der Ein$\mathrm{HluB}$ bei kürzeren Bögen geringer ausfallen muß wie bei längeren, wenn man setzt:

$$
t^{\prime}=\frac{t}{1-\gamma t},
$$

wo $t^{\prime}$ die korrigierte mittlere Aufenthaltsdaver eines Teilchens im Bogen ist, und $\gamma$ eine Konstante bedeutet. Hiermit fände sich dann unsere frühere Formel (3). $\left.{ }^{1}\right)$ Ob die Annahme, daB nur der Beschleunigung proportionale Widerstände sich der Bewegung der Teilchen eventuell entgegenstellen, zulässig ist, wird noch der Revision bedürfen, sie würde sich eventuell auf eine Mitführung des Mediums gründen lassen.

\section{\$ 4. Verteilung der Strahlung auf die einzelnen Teile des Bogens.}

Aus der im Paragraph 2 festgestellten Unabhängigkeit der lichtelektrischen Strahlung von der Richtung wurde der SchluB gezogen, da $B$ diese Strablung vom Bogen herrühre. $\mathrm{Um}$ diesen Schluß einwandfrei zu machen und gleichzeitig zu sehen, $o b$ die Strahlung nicht etwa auf einzelne Teile des

1) Anm. bei der Korrektur. Es hat sich inzwischen ergeben, dab die Berücksichtigrung der Spannungsverluste das Glied 0,058 p.56 Formel(3) wahrscheinlich zum Versehwinden bringt. Hierzu sind weitere Versuche im Gang, da bei den früheren jene Verluste nicht genügend genau und häufig bestimmt worden waren. 
Bogens beschränkt sei, sondern sich auf den ganzen Bogen erstrecke und zwar in Konsequenz der am SchluB des vorigen Paragraphen entwickelten Anschauung mit abnehmender Stärke von der positiven nach der negativen Kohle hin, gelangten noch einige Versuche zur Ausführung, bei welchen eine Blende die Strahlung der einzelnen Bogenteile aussonderte.

Dabei wurde folgende Anordnung benutzt. Die Blende, ein Glimmerblatt, stand so dicht als angängig, etwa $1,5 \mathrm{~cm}$, herangerückt mit horizontaler Kante vor dem vertikalen Bogen. lhre Befestigung an einem durch Zahnstange und Trieb ver. tikal verschiebbaren Halter gestattete die Blende folgeweise so zu stellen, daB sie erst den Bogen frei lieb und ihn dann von der positiven Kohle her schrittweise abblendete. Auf gleicher Höhe mit dem Bogen in etwa $90 \mathrm{~cm}$ Abstand befand sich die vertikal nur $2 \mathrm{~cm}$ hohe, horizontal $12 \mathrm{~cm}$ lange lichtelektrische Zelle. Unter diesen Verhältnissen erhielten die verschiedenen Punkte der Zelle von bis auf verschwindende Beträge gleichen Bogenteilen Strahlung. Die Beobachtung der abgeblendeten Bogenteile gestattete eine möglichst nahe hinter der Zelle angebrachte Linse, welche bis auf zwei schmale, horizontale Spalten, gerade über und unter dem Rand der bestrahlten Platte, abgeblendet war und auf einen entfernt stehenden Schirm vom Bogen und der Glimmerblende ein etwa fünffach vergrößertes Bild entwarf. Auf demselben hatte gemäB der getroffenen Anordnung der Rand der Blende dieselbe Lage zum Bogen, wie für das von der Zelle direkt nach dem Bogen blickende Auge. Die Umrisse des Bogens und der Kohlen, sowie der Rand der Blende wurden auf dem Schirm mit Blei nachgefahren und so die Figuren 2 erhalten.

Die folgende Tabelle 12 liefert die Ergebnisse der mit dieser Anordnung angestellten Versuche. In der ersten Kolumne sind die Ladungszeiten $\tau$ enthalten, und zwar im allgemeinen je zwei Werte, da die Blende nach Erreichung ihrer höchsten lage wieder zurückgeführt und bei den vorherigen Stellungen derselben je eine zweite Beobachtung ausgeführt wurde. Ihre Mittel $\bar{\tau}$ stehen in Kolumne 2. Die dritte Kolumne gibt die zugehörigen Mittelwerte der Stromstärke, die vierte die, soweit erforderlich, auf 19,5 Amp. reduzierten Werte von $\vec{\tau}$, die fünfte die der Strahlungsintensität $s$ proportionalen 
Werte $1000 / \overline{\boldsymbol{\tau}}_{r}$, die sechste die am Projektionsbild, und zwar von der Kathode ab gemessenen Längen $l$ des nicht abgeblendeten Bogenteiles. Durch Subtraktion aufeinander folgender Werte von $l$ bez. $s$ ergaben sich die in Kolumne 7 und 8 ent-
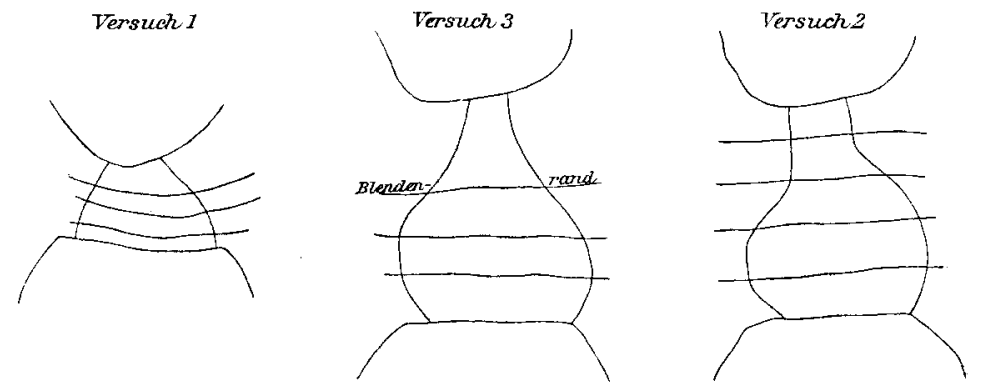

Fig. 2.

haltenen Längen $l^{\prime}$ der einzelnen Bogenschichten und der von ihnen ausgehenden Strahlungsintensitäten $\boldsymbol{s}^{\prime}$, wobei die Werte in der Richtung Anode-Kathode aufeinander folgen. Aus Kolumne 9 ist das mittlere Strablungsvermögen der einzelnen Schichten zu entnehmen.

Tabelle 12.

\begin{tabular}{|c|c|c|c|c|c|c|c|c|c|c|}
\hline $\begin{array}{l}\text { Ver- } \\
\text { such }\end{array}$ & \multicolumn{2}{|c|}{$\begin{array}{c}t \\
\text { sec }\end{array}$} & $\begin{array}{c}\vec{\tau} \\
\sec \end{array}$ & $\begin{array}{c}i \\
\text { Amp. }\end{array}$ & $\begin{array}{c}\bar{t}_{r} \\
(19,5 \text { A.) }\end{array}$ & $s=\frac{1000}{\overrightarrow{\boldsymbol{t}}_{r}}$ & $\begin{array}{c}l \\
\mathrm{~mm}\end{array}$ & $\begin{array}{c}l^{\prime} \\
\mathrm{mm}\end{array}$ & $s^{\prime}$ & $\frac{s^{\prime}}{l^{\prime}}$ \\
\hline \multirow{4}{*}{1} & 17,5 & 19 & 18,2 & 19,65 & 18,4 & 54,9 & 27 & 4 & 19,4 & 4,8 \\
\hline & 29 & 27,5 & 28,2 & 19,55 & 28,3 & 35,5 & 23 & 7 & 15,6 & 2,25 \\
\hline & 50 & 50,5 & 50,2 & 19,5 & 50,2 & 19,9 & 16 & 7 & 11,3 & 1,61 \\
\hline & \multicolumn{2}{|c|}{115} & 115 & 19,55 & 115 & 8,6 & 9 & 9 & 8,6 & 0,96 \\
\hline \multirow{5}{*}{2} & 13 & 14 & 13,5 & 18,5 & 12,5 & 80 & 67 & 13,5 & 31,2 & 2,31 \\
\hline & 22 & 21 & 21,5 & 18,4 & 20,5 & 48,8 & 53,5 & 13,5 & 23,2 & 1,72 \\
\hline & 42,5 & 42 & 42,2 & 18,5 & 39 & 25,6 & 40 & 13 & 15,2 & 1,15 \\
\hline & 102,5 & 106 & 104 & 18,8 & 96 & 10,4 & 27 & 13,5 & 6,3 & 0,47 \\
\hline & 26 & & 261 & 18,4 & 243 & 4,1 & 13,5 & 13,5 & 4,1 & 0,30 \\
\hline \multirow[b]{4}{*}{. } & 13 & 13 & 13 & 19,2 & 12,8 & 78 & 66,5 & 13,5 & 32,4 & 2,40 \\
\hline & 23,5 & 22 & 22,8 & 19,0 & 21,9 & 45,7 & 53 & 13 & 19,4 & 1,49 \\
\hline & 42,5 & 38,5 & 40,5 & 18,6 & 38,0 & 26,3 & 40 & 13,5 & 15,7 & 1,16 \\
\hline & 9 & & 99 & 18,8 & 94 & 10,6 & 26,5 & 26,5 & 10,6 & 0,40 \\
\hline
\end{tabular}


Aus diesen Beobachtungen ergibt sich, daß der Bogen seiner ganzen Länge nach an der Strahlung teil nimmt und zwar im Einklang mit dem Schluß von $\& 3$ von der Anode nach der Kathode hin mit abnehmender Stärke. Rückte man in Fortsetzung des Versuches 1, Tab. 12, die Blende so, daB sie die Anode und von dieser her den ganzen Bogen bedeckte und gerade eben die Kathode berührte, so ergaben sich 490 Sek. für $\tau$, d. h. nicht ganz 4 Proz. der gesamten Strablung kamen aus dem Kathodengebiet. Die Kathode ist also an der Strahlung nur minimal oder vielleicht auch gar nicht beteiligt, indem ja die strablenden Partikelchen teilweise auch erst jenseits der durch den Blendenrand bezeichneten Berührungsebene der Kathode die letztere treffen.

Bekam im AnschluB an Versuch 2 die Blende eine solche Lage, daB sie die Kathode und von dieser her den Bogen fast ganz bedeckte und mit ihrem Rand die äubersten Spitzen der positiven Kohle berührte, wobei wegen der gerade vorbandenen Krümmung der Randprojektion noch eine kleine Spur des Bogens und, falls die Zelle etwa von einem äußersten Streifchen des Kraters noch bestrahlt werden konnte, auch dieses frei blieb, so betrug $\tau 149$ Sek. Etwa 8 Proz. der Strablung kamen also von dem noch frei bleibenden Teile. Da nun nach dem Verlauf von $s^{\prime} / l^{\prime}$, wie er sich aus der 'Tabelle ergibt, die ersten 8 Proz. der Strahlung von einer nur ca. 0,4 mm dicken, der Anode anliegenden Bogenschicht kommen mußten, so viel aber etwa von der Blende ungefähr noch frei gelassen wurde, so folgt, daB auch die Anode an der lichtelektrischen Strahlung jedenfalls nur minimal beteiligt sein kann. Aus allem zusammengenommen ergibt sich, daß diese Strahlung vom Bogen ausgeht, die Elektroden dazu jedenfalls nur einen sehrkleinen, eventuell gar keinen Beitrag liefern, wodurch die früheren Schlüsse in dieser Richtung bestätigt werden.

Die Strahlung des $27 \mathrm{~mm}$ langen Bogens (Versuch 1, Tabelle 12) war 55, diejenige der ersten 27 bez. $26,5 \mathrm{~mm}$ des 67 bez. 66,5 mm langen Bogens (Versuch 2 und 3) $\mathbf{5 4}$ bez. 52. Die beiden Strahlungen ergaben sich also innerhalb der erzielten Genauigkeitsgrenzen als gleich, wodurch eine Konsequenz des am SchluB des $\S 3$ Entwickelten ihre quantitative Bestätigung findet. Man könnte v. a. auch annehmen, 
daB die Teilchen beim Durchlaufen des Bogens immer mehr an Strahlungsvermögen einbüßen, wäre dann aber, um der Formel (3) zu genügen, zu der Annahme gezwungen, daß das Strahlungsvermögen der Wurzel aus dem Abstand von der Anode umgekehrt proportional ist. Demgegenüber ist die oben entwickelte Anschaung doch so einfach, daB man sich einer weiteren Prüfung derselben, trotz mancher Bedenken, welche sich zur Zeit noch entgegenstellen, nicht wird entziehen können.

Vergleicht man mit der eben besprochenen Strahlung der ersten $27 \mathrm{~mm}$ diejenige der zweiten $27 \mathrm{~mm}$, wofür Versuch 2 Beobachtungen enthält, so ist das Verhältnis

$$
\frac{s_{2}}{s_{1}}=\frac{21,7}{54}=0,40,
$$

wonach sich das oben für den ganzen Bogen gefundene, angenäherte Wurzelgesetz auch für einzelne Abschnitte des Bogens bestätigt, da nach ihm $s_{2} / s_{1}=\sqrt{2}-1$ sein sollte.

Um eine derartige Vergleichung auf alle einzelnen Schichten ausàehnen zu können, sind in der folgenden Tabelle 13 die aus den Beobachtungen direkt durch Subtraktion gefundenen Werte $s^{\prime}$ (vgl. Tabelle 12) mit Werten $s^{\prime \prime}$ zusammengestellt, welche nach der Formel

$$
s^{\prime \prime}=C\left[\sqrt{l_{e}}-\sqrt{l_{a}}\right]
$$

berechnet wurden, wo $l_{e}$ und $l_{a}$ die Strecken des Bogens von der Anode bis zum Ende bez. Anfang der betrachteten Schicht bedeuten. $\boldsymbol{s}^{\prime}$ und $\boldsymbol{s}^{\prime \prime}$ sollten annähernd gleich verlaufen und zwar nur annähernd: erstens, weil die Potentialdifferenzen der einzelnen Schichten nicht deren Dicke proportional zu sein brauchen, wenn auch die ganze Bogenlänge der gesamten auf den Bogen entfallenden Potentialdifferenz nahezu proportional ist, zweitens, weil die Werte $l_{e}$ und $l_{a}$ etwas unter der Zartheit des Blendenrandbildes litten, was sich wegen der in der Formel vorkommenden Differenz stärker geltend machen muB, drittens, wegen der in der Formel für $s^{\prime \prime}$ nicht berücksichtigten, im $\S 3$ erwähnten Krümmung der Bahnen. 
Tabelle 13.

\begin{tabular}{|c|c|c|c|c|c|c|c|c|c|c|c|c|c|}
\hline \multirow[b]{2}{*}{$\begin{array}{l}\text { Bogen- } \\
\text { strecke }\end{array}$} & \multicolumn{4}{|c|}{ Versuch 1} & \multicolumn{5}{|c|}{ Versuch 2} & \multicolumn{4}{|c|}{ Versuch 3} \\
\hline & $\begin{array}{c}0 \\
\text { bis } \\
4\end{array}$ & $\begin{array}{c}4 \\
\text { bis } \\
11\end{array}$ & $\begin{array}{r}11 \\
\text { bis } \\
18 \\
\end{array}$ & $\begin{array}{r}18 \\
\text { bis } \\
27\end{array}$ & $\begin{array}{c}0 \\
\text { bis } \\
13,5\end{array}$ & \begin{tabular}{|c}
13,5 \\
bis \\
27
\end{tabular} & $\begin{array}{r}27 \\
\text { bis } \\
40\end{array}$ & $\begin{array}{c}40 \\
\text { bis } \\
53,5\end{array}$ & $\begin{array}{c}53,5 \\
\text { bis } \\
67\end{array}$ & $\begin{array}{c}0 \\
\text { bis } \\
13,5\end{array}$ & $\left|\begin{array}{c}13,5 \\
\text { bis } \\
26,5\end{array}\right|$ & $\mid \begin{array}{c}26,5 \\
\text { bis } \\
40\end{array}$ & $\begin{array}{c}40 \\
\text { bis } \\
67 \\
\end{array}$ \\
\hline$s^{\prime}$ & 19,4 & 15,6 & 11,3 & 8,6 & 31,2 & 23,2 & 15,2 & 6,3 & 4,1 & 32,4 & 19,4 & 15,7 & 10,6 \\
\hline$s^{\prime \prime}$ & 20,0 & 13,1 & 8,3 & 9,6 & 36,7 & 15,3 & 11,2 & 9,9 & 8,7 & 36,7 & 14,8 & 11,7 & 18,3 \\
\hline
\end{tabular}

Allen drei Versuchen liegt derselbe Wert von $C$ zugrunde, für den sich als bestes Mittel aus sämtlichen Versuchen 10,0 ergab. Unter Berücksichtigung des oben Gesagten ist der Anschluß der berechneten an die beobachteten Werte genügend. Nach den Zahlen der Tabelle scheinen die Geschwindigkeiten der Teilchen in der Nähe der Elektroden über, in der Mitte unter dem Wert zu liegen, welcher bei gleichförmiger "Beschleunigung erhalten würde, beim langen Bogen tritt dies stärker hervor wie beim kurzen und an der Kathode stärker wie an der Anode. Die Annahme einer dementsprechenden Feldverteilung bietet keine Schwierigkeit, eventuell macht sich auch in der Nähe der Kathode die negative Entladung geltend.

Zum SchluB möge noch bemerkt werden, daB die Prüfung anderer Ansätze nicht zum Ziel führte; u. a. war z. B. zu prüfen, ob die Strahlung etwa dem Volumen $v^{\prime}$ der einzelnen Bogenschichten oder Bögen proportional wäre. Berechnete man die $v^{\prime}$ aus der Figur 2 unter Annahme des Bogens als Rotationskörper und bildete für alle Versuche die Werte $s^{\prime} / v^{\prime}$, so variierten diese zwischen 0,19 und 1,21 , so dab also keine Proportionalität besteht.

Bei der vorliegenden Untersuchung wurden als Anoden Dochtkohlen verwendet. Ob die strahlenden Teilchen wesentlich dem Docht entstammen, mögen spätere Versuche entscheiden.

Dresden, Phys. Inst. d. Techn. Hochschule, Oktober 1903.

(Eingegangen 17. Oktober 1903.) 